\title{
GENERALIZED ORDER STATISTICS FROM KUMARASWAMY-BURR III DISTRIBUTION AND RELATED INFERENCE
}

\author{
B. SINGH, R. U. KHAN and M. A. R. KHAN \\ Department of Statistics and Operations Research \\ Aligarh Muslim University \\ Aligarh-202002 \\ India \\ e-mail: aruke@rediffmail.com
}

\begin{abstract}
In this paper, some recurrence relations for single and product moments of generalized order statistics arising from the Kumaraswamy-Burr III distribution are obtained. Further, various deductions and related results are discussed and identified some of these recurrence relations and other properties of generalized order statistics which characterize this distribution.
\end{abstract}

\section{Introduction}

The concept of generalized order statistics (gos) was given by Kamps [13], which is given as below:

Let $X_{1}, X_{2}, \ldots, X_{n}$ be a sequence of independent and identically distributed (iid) random variables ( $r v$ ) with absolutely continuous

2010 Mathematics Subject Classification: 62G30, 62E10.

Keywords and phrases: order statistics, record values, generalized order statistics, Kumaraswamy-Burr III distribution, single moments, product moments, recurrence relations, conditional expectation and characterization.

Received December 26, 2017

(ㄷ) 2018 Scientific Advances Publishers 
distribution function $(d f) F(x)$ and the probability density function ( $p d f)$ $f(x), x \in(\alpha, \beta)$. Let $n \in N, n \geq 2, k \geq 1, \tilde{m}=\left(m_{1}, m_{2}, \ldots, m_{n-1}\right) \in \mathfrak{R}^{n-1}$, $M_{r}=\sum_{j=r}^{n-1} m_{j}, 1 \leq r \leq n-1$, be the parameters such that $\gamma_{r}=k+n-r$ $+M_{r} \geq 1$, for all $r \in\{1,2, \ldots, n-1\}$. Then $X(r, n, \tilde{m}, k), r=1,2, \ldots, n$ are called gos if their joint $p d f$ is given by

$$
\begin{aligned}
f_{X(1, n, \tilde{m}, k), \ldots, X}(n, n, \tilde{m}, k) & \left(x_{1}, x_{2}, \ldots, x_{n}\right) \\
= & k\left(\prod_{j=1}^{n-1} \gamma_{j}\right)\left(\prod_{i=1}^{n-1}\left[\bar{F}\left(x_{i}\right)\right]^{m_{i}} f\left(x_{i}\right)\right)\left[\bar{F}\left(x_{n}\right)\right]^{k-1} f\left(x_{n}\right)
\end{aligned}
$$

on the cone $F^{-1}(0)<x_{1} \leq \ldots \leq x_{n}<F^{-1}(1)$, where $\bar{F}(x)=1-F(x)$.

Here we may consider two cases:

Case. (i) $\gamma_{i}=\gamma_{j}$, i.e., $m_{1}=m_{2}=\ldots=m_{n-1}=m$.

Case. (ii) $\gamma_{i} \neq \gamma_{j}, i, j=1,2, \ldots, n-1$.

For case I, gos will be denoted as $X(r, n, m, k)$ and its $p d f$ is given by (Kamps [13])

$$
f_{X(r, n, m, k)}(x)=\frac{C_{r-1}}{(r-1) !}[\bar{F}(x)]^{\gamma_{r}-1} f(x) g_{m}^{r-1}(F(x)), \alpha \leq x \leq \beta,
$$

and the joint $p d f$ of $X(r, n, m, k)$ and $X(s, n, m, k), 1 \leq r<s \leq n$, is

$$
\begin{aligned}
f_{X(r, n, m, k), X(s, n, m, k)}(x, y)= & \frac{C_{s-1}}{(r-1) !(s-r-1) !}[\bar{F}(x)]^{m} f(x) g_{m}^{r-1}(F(x)) \\
& \times\left[h_{m}(F(y))-h_{m}(F(x))\right]^{s-r-1}[\bar{F}(y)]^{\gamma_{s}-1} f(y), \\
& \alpha \leq x<y \leq \beta
\end{aligned}
$$


where

$$
\begin{aligned}
C_{r-1} & =\prod_{i=1}^{r} \gamma_{i}, \quad \gamma_{i}=k+(n-i)(m+1), \\
h_{m}(x) & = \begin{cases}-\frac{1}{m+1}(1-x)^{m+1}, & m \neq-1, \\
-\ln (1-x), & m=-1,\end{cases}
\end{aligned}
$$

and

$$
g_{m}(x)=h_{m}(x)-h_{m}(0), \quad x \in[0,1) .
$$

For case II, the $p d f$ of $X(r, n, \tilde{m}, k)$ is (Kamps and Cramer [14])

$$
f_{X(r, n, \tilde{m}, k)}(x)=C_{r-1} f(x) \sum_{i=1}^{r} a_{i}(r)[\bar{F}(x)]^{\gamma_{i}-1},
$$

and the joint $p d f$ of $X(r, n, \tilde{m}, k)$ and $X(s, n, \tilde{m}, k), 1 \leq r<s \leq n$, is

$$
\begin{aligned}
f_{X(r, n, \tilde{m}, k) X(s, n, \tilde{m}, k)}(x, y)= & C_{s-1} \sum_{i=r+1}^{s} a_{i}^{(r)}(s)\left(\frac{\bar{F}(y)}{\bar{F}(x)}\right)^{\gamma_{i}}\left(\sum_{i=1}^{r} a_{i}(r)[\bar{F}(x)]^{\gamma_{i}}\right) \\
& \times \frac{f(x)}{\bar{F}(x)} \frac{f(y)}{\bar{F}(y)}
\end{aligned}
$$

where

$$
\begin{aligned}
C_{r-1} & =\prod_{i=1}^{r} \gamma_{i}, \quad \gamma_{i}=k+n-i+M_{i}, \\
a_{i}(r) & =\prod_{\substack{j=1 \\
j \neq i}}^{r} \frac{1}{\left(\gamma_{j}-\gamma_{i}\right)}, \quad 1 \leq i \leq r \leq n, \\
a_{i}^{(r)}(s) & =\prod_{\substack{j=r+1 \\
j \neq i}}^{s} \frac{1}{\left(\gamma_{j}-\gamma_{i}\right)}, \quad r+1 \leq i \leq s \leq n .
\end{aligned}
$$


It may be noted that $m_{1}=m_{2}=\ldots=m_{n-1}=m \neq-1$

$$
\begin{aligned}
& a_{i}(r)=\frac{(-1)^{r-i}}{(r-1) !(m+1)^{r-1}}\left(\begin{array}{c}
r-1 \\
r-i
\end{array}\right), \\
& a_{i}^{(r)}(s)=\frac{(-1)^{s-i}}{(s-r-1) !(m+1)^{s-r-1}}\left(\begin{array}{c}
s-r-1 \\
s-i
\end{array}\right) .
\end{aligned}
$$

Consequently, the $p d f$ of $X(r, n, \tilde{m}, k)$ and the joint $p d f$ of $X(r, n, \tilde{m}, k)$ and $X(s, n, \tilde{m}, k)$ reduce to the $p d f$ of $X(r, n, m, k)$ and the joint $p d f$ of $X(r, n, m, k)$ and $X(s, n, m, k)$, respectively.

Several models of ordered random variables such as order statistics and record value can be seen as special cases of gos. If $m=0$ and $k=1$, then $X(r, n, m, k)$ reduces to the $r$-th order statistic $X_{r: n}$ (David and Nagaraja [8]). If $m=-1$ and $k=1$, then $X(r, n, m, k)$ is the $r$-th record values from an infinite sequence of iid ru's (Ahsanullah [2]). Other special cases are $k$-th record values ( $m=-1$, Dziubdziela and Kopociński [9]) and order statistics with non-integral sample size $(m=0, k=\alpha-n+1, \alpha=n$, Stigler [24], Rohatgi and Saleh [23]).

Many authors utilized the concept of gos in their studies. References may be made to Kamps and Gather [15], Keseling [16], Cramer and Kamps [6], Ahsanullah [3], Habibullah and Ahsanullah [10], Ahmad and Fawzy [1], Bieniek and Szynal [5], Cramer et al. [7], Khan and Alzaid [17], Khan et al. [18], Khan et al. [21], Khan and Zia [20], Khan and Khan [19] among others. In this paper, we mainly focus on the study of gos arising from the Kumaraswamy-Burr III distribution.

A random variable $X$ is said to have Kumaraswamy-Burr III distribution (Huang and Oluyede [11]) if its $p d f$ is of the form

$$
f(x)=\alpha \beta \lambda x^{-\beta-1}\left(1+x^{-\beta}\right)^{-\alpha-1}\left(1-\left(1+x^{-\beta}\right)^{-\alpha}\right)^{\lambda-1}, \quad x>0, \quad \alpha, \beta, \lambda>0,
$$


with the corresponding $d f$

$$
F(x)=1-\left[1-\left(1+x^{-\beta}\right)^{-\alpha}\right]^{\lambda}, \quad x>0, \quad \alpha, \beta, \lambda>0 .
$$

It is easy to see that

$$
\alpha \beta \lambda \bar{F}(x)=\left\{\alpha x+\sum_{u=2}^{\alpha+1}\left(\begin{array}{c}
\alpha+1 \\
u
\end{array}\right) x^{\beta(1-u)+1}\right\} f(x) .
$$

\section{Relations for Single Moments}

Theorem 2.1. For the distribution given in (1.7) and $n \in N, \tilde{m} \in \mathfrak{R}$, $2 \leq r \leq n, j=0,1,2, \ldots$

$$
\begin{aligned}
\left(1-\frac{j}{\beta \lambda \gamma_{r}}\right) E\left[X^{j}(r, n, \tilde{m}, k)\right]=E\left[X^{j}(r-1, n, \tilde{m}, k)\right] \\
+\frac{j}{\alpha \beta \lambda \gamma_{r}} \sum_{u=2}^{\alpha+1}\left(\begin{array}{c}
\alpha+1 \\
u
\end{array}\right) E\left[X^{j+\beta(1-u)}(r, n, \tilde{m}, k)\right] .
\end{aligned}
$$

Proof. For $2 \leq r \leq n, n \geq 2$ and $k=1,2, \ldots$, we have Athar and Islam [4]

$$
\begin{aligned}
E[\xi(X(r, n, \tilde{m}, k))]- & E[\xi(X(r-1, n, \tilde{m}, k))] \\
& =C_{r-2} \int_{\alpha}^{\beta} \xi^{\prime}(x) \sum_{i=1}^{r} a_{i}(r)[\bar{F}(x)]^{\gamma_{i}} d x,
\end{aligned}
$$

where $\xi(x)$ is a measurable function of $x$ and is differentiable.

Let $\xi(x)=x^{j}$, then

$E\left[X^{j}(r, n, \tilde{m}, k)\right]-E\left[X^{j}(r-1, n, \tilde{m}, k)\right]=j C_{r-2} \int_{0}^{\infty} x^{j-1} \sum_{i=1}^{r} a_{i}(r)[\bar{F}(x)]^{\gamma_{i}} d x$. 
On using (1.6) in (2.3), we find that

$$
\begin{aligned}
& E\left[X^{j}(r, n, \tilde{m}, k)\right]-E\left[X^{j}(r-1, n, \tilde{m}, k)\right] \\
& =\frac{j}{\alpha \beta \lambda \gamma_{r}}\left(\alpha C_{r-1} \int_{0}^{\infty} x^{j} f(x) \sum_{i=1}^{r} a_{i}(r)[\bar{F}(x)]^{\gamma_{i}-1} d x\right) \\
& \quad+\frac{j}{\alpha \beta \lambda \gamma_{r}} \sum_{u=2}^{\alpha+1}\left(\begin{array}{c}
\alpha+1 \\
u
\end{array}\right)\left(C_{r-1} \int_{0}^{\infty} x^{j+\beta(1-u)} f(x) \sum_{i=1}^{r} a_{i}(r)[\bar{F}(x)]^{\gamma_{i}-1} d x\right),
\end{aligned}
$$

which lead to (2.1).

Corollary 2.1. For $m_{1}=m_{2}=\ldots=m_{n-1}=m \neq-1$, the recurrence relation for single moments of gos from Kumaraswamy-Burr III distribution has the form

$$
\begin{aligned}
&\left(1-\frac{j}{\beta \lambda \gamma_{r}}\right) E\left[X^{j}(r, n, m, k)\right] \\
&=E\left[X^{j}(r-1, n, m, k)\right] \\
&+\frac{j}{\alpha \beta \lambda \gamma_{r}} \sum_{u=2}^{\alpha+1}\left(\begin{array}{c}
\alpha+1 \\
u
\end{array}\right) E\left[X^{j+\beta(1-u)}(r, n, m, k)\right] .
\end{aligned}
$$

Remark 2.1. Recurrence relation for single moments of order statistics (at $m=0, k=1$ ) from Kumaraswamy-Burr III distribution is

$$
\left(1-\frac{j}{\beta \lambda(n-r+1)}\right) E\left(X_{r: n}^{j}\right)=E\left(X_{r-1: n}^{j}\right)+\frac{j}{\alpha \beta \lambda(n-r+1)} \sum_{u=2}^{\alpha+1}\left(\begin{array}{c}
\alpha+1 \\
u
\end{array}\right) E\left(X_{r: n}^{j+\beta(1-u)}\right) .
$$

Remark 2.2. The recurrence relation for single moments of $k$-th record values will be

$$
\begin{aligned}
\left(1-\frac{j}{\beta \lambda k}\right) E\left[X^{j}(r, n,-1, k)\right]= & E\left[X^{j}(r-1, n,-1, k)\right] \\
& +\frac{j}{\alpha \beta \lambda k} \sum_{u=2}^{\alpha+1}\left(\begin{array}{c}
\alpha+1 \\
u
\end{array}\right) E\left[X^{j+\beta(1-u)}(r, n,-1, k)\right],
\end{aligned}
$$


or

$$
\left(1-\frac{j}{\beta \lambda k}\right) E\left(X_{U_{r}}^{(k)}\right)^{j}=E\left(X_{U_{r-1}}^{(k)}\right)^{j}+\frac{j}{\alpha \beta \lambda k} \sum_{u=2}^{\alpha+1}\left(\begin{array}{c}
\alpha+1 \\
u
\end{array}\right) E\left(X_{U_{r}}^{(k)}\right)^{j+\beta(1-u)},
$$

where $X_{U_{r}}^{(k)}, r=1,2, \ldots$ is $r$-th $k$ records, which verify the result obtained by Kumar et al. [22].

For upper records $(k=1)$, the recurrence relation for single moments from Kumaraswamy-Burr III distribution is deduced as obtained by Kumar et al. [22].

\section{Relations for Product Moments}

Theorem 3.1. For distribution given in (1.7) and for $k, n \in N$, $\tilde{m}=\left(m_{1}, m_{2}, \ldots, m_{n-1}\right) \in \mathfrak{R}, 1 \leq r<s \leq n-1$ and $i, j \geq 0$.

$$
\begin{aligned}
(1- & \left.\frac{j}{\beta \lambda \gamma_{s}}\right) E\left[X^{i}(r, n, \tilde{m}, k) X^{j}(s, n, \tilde{m}, k)\right] \\
= & E\left[X^{i}(r, n, \tilde{m}, k) X^{j}(s-1, n, \tilde{m}, k)\right] \\
& \quad+\frac{j}{\alpha \beta \lambda \gamma_{s}} \sum_{u=2}^{\alpha+1}\left(\begin{array}{c}
\alpha+1 \\
u
\end{array}\right) E\left[X^{i}(r, n, \tilde{m}, k) X^{j+\beta(1-u)}(s, n, \tilde{m}, k)\right] .
\end{aligned}
$$

Proof. In view of Athar and Islam [4], note that

$$
\begin{gathered}
E[\xi\{X(r, n, \tilde{m}, k), X(s, n, \tilde{m}, k)\}]-E[\xi\{X(r, n, \tilde{m}, k), X(s-1, n, \tilde{m}, k)\}] \\
=C_{s-2} \int_{0}^{\infty} \int_{x}^{\infty} \xi^{\prime}(x, y) \sum_{i=r+1}^{s} a_{i}^{(r)}(s)\left(\frac{\bar{F}(y)}{\bar{F}(x)}\right)^{\gamma_{i}}\left(\sum_{i=1}^{r} a_{i}(r)[\bar{F}(x)]^{\gamma_{i}}\right) \frac{f(x)}{\bar{F}(x)} d y d x,
\end{gathered}
$$


Let

$$
\begin{gathered}
\xi(x, y)=x^{i} y^{j}, \text { then } \\
E\left[X^{i}(r, n, \tilde{m}, k) X^{j}(s, n, \tilde{m}, k)\right]-E\left[X^{i}(r, n, \tilde{m}, k) X^{j}(s-1, n, \tilde{m}, k)\right] \\
=j C_{s-2} \int_{0}^{\infty} \int_{x}^{\infty} x^{i} y^{j-1} \sum_{i=r+1}^{s} a_{i}^{(r)}(s)\left(\frac{\bar{F}(y)}{\bar{F}(x)}\right)^{\gamma}\left(\sum_{i=1}^{r} a_{i}(r)[\bar{F}(x)]^{\gamma_{i}}\right) \frac{f(x)}{\bar{F}(x)} d y d x .
\end{gathered}
$$

On substituting (1.8) in (3.3), we get

$$
\begin{aligned}
& E\left[X^{i}(r, n, \tilde{m}, k) X^{j}(s, n, \tilde{m}, k)\right]-E\left[X^{i}(r, n, \tilde{m}, k) X^{j}(s-1, n, \tilde{m}, k)\right] \\
& =\frac{j}{\beta \lambda \gamma_{s}}\left[C_{s-1} \int_{0}^{\infty} \int_{x}^{\infty} x^{i} y^{j} \sum_{i=r+1}^{s} a_{i}^{(r)}(s)\left(\frac{\bar{F}(y)}{\bar{F}(x)}\right)^{\gamma_{i}}\left(\sum_{i=1}^{r} a_{i}(r)[\bar{F}(x)]^{\gamma_{i}}\right) \frac{f(x) f(y)}{\bar{F}(x) \bar{F}(y)} d y d x\right. \\
& +\frac{j}{\alpha \beta \lambda \gamma_{s}} \sum_{u=2}^{\alpha+1}\left(\begin{array}{c}
\alpha+1 \\
u
\end{array}\right) C_{s-1} \int_{0}^{\infty} \int_{x}^{\infty} x^{i} y^{j+\beta(1-u)} \sum_{i=r+1}^{s} a_{i}^{(r)}(s)\left(\frac{\bar{F}(y)}{\bar{F}(x)}\right)^{\gamma_{i}} \\
& \left.\quad \times\left(\sum_{i=1}^{r} a_{i}(r)[\bar{F}(x)]^{\gamma_{i}}\right) \frac{f(x) f(y)}{\bar{F}(x) \bar{F}(y)} d y d x\right],
\end{aligned}
$$

which after simplification yields (3.1).

At $i=0$ in (3.1), the recurrence relation for product moments reduces to relation for single moments as obtained in (2.1).

Corollary 3.1. For $m_{1}=m_{2}=\ldots=m_{n-1}=m \neq-1$, the recurrence relation for product moments of gos from Kumaraswamy-Burr III distribution has the form

$$
\begin{aligned}
\left(1-\frac{j}{\beta \lambda \gamma_{s}}\right) & E\left[X^{i}(r, n, m, k) X^{j}(s, n, m, k)\right] \\
= & E\left[X^{i}(r, n, m, k) X^{j}(s-1, n, m, k)\right] \\
& +\frac{j}{\alpha \beta \lambda \gamma_{s}} \sum_{u=2}^{\alpha+1}\left(\begin{array}{c}
\alpha+1 \\
u
\end{array}\right) E\left[X^{j+\beta(1-u)}(r, n, m, k)\right] .
\end{aligned}
$$


Remark 3.1. Recurrence relation for product moments of order statistics (at $m=0, k=1$ ) from Kumaraswamy-Burr III distribution is

$$
\begin{aligned}
& \left(1-\frac{j}{\beta \lambda(n-s+1)}\right) E\left(X_{r: n}^{i} X_{s: n}^{j}\right) \\
& \quad=E\left(X_{r: n}^{i} X_{s-1: n}^{j}\right)+\frac{j}{\alpha \beta \lambda(n-s+1)} \sum_{u=2}^{\alpha+1}\left(\begin{array}{c}
\alpha+1 \\
u
\end{array}\right) E\left(X_{r: n}^{i} X_{s: n}^{j+\beta(1-u)}\right) .
\end{aligned}
$$

Remark 3.2. Setting $m=-1$ and $k \geq 1$ in (3.1), we deduce the recurrence relation for product moments of $k$-th upper record values from the Kumaraswamy-Burr III distribution, established by Kumar et al. [22]).

\section{Characterizations}

Let $X(r, n, m, k), r=1,2, \ldots, n$ be gos, then the conditional $p d f$ of $X(s, n, m, k)$ given $X(r, n, m, k)=x, 1 \leq r<s \leq n$, is

$$
\begin{aligned}
f_{X(s, n, m, k) \mid X(r, n, m, k)}(y \mid x) \\
=\frac{C_{s-1}}{(s-r-1) ! C_{r-1}}[\bar{F}(x)]^{m-\gamma_{r}+1} \\
\quad \times\left[h_{m}(F(y))-h_{m}(F(x))\right]^{s-r-1}[\bar{F}(y)]^{\gamma_{s}-1} f(y), \quad x<y .
\end{aligned}
$$

Theorem 4.1. Let $X$ be a non-negative random variable having an absolutely continuous $d f F(x)$ with $F(0)=0$ and $0 \leq F(x) \leq 1$ for all $x>0$, then

$E[\xi\{X(s, n, m, k)\} \mid X(l, n, m, k)=x]$

$$
=\left[1-\left(1+x^{-\beta}\right)^{-\alpha}\right]^{\lambda} \prod_{j=1}^{s-l}\left(\frac{\gamma_{l+j}}{\gamma_{l+j}+1}\right), \quad l=r, r+1,
$$

if and only if

$$
\bar{F}(x)=\left[1-\left(1+x^{-\beta}\right)^{-\alpha}\right]^{\lambda}, \quad x>0, \alpha, \beta, \lambda>0,
$$


where

$$
\xi(y)=\left[1-\left(1+y^{-\beta}\right)^{-\alpha}\right]^{\lambda} .
$$

Proof. From (4.1) for $s>r+1$, we have

$$
\begin{aligned}
E[ & \xi\{(s, n, m, k)\} \mid X(r, n, m, k)=x] \\
= & \frac{C_{s-1}}{(s-r-1) ! C_{r-1}(m+1)^{s-r-1}} \\
& \times \int_{x}^{\infty}\left[1-\left(1+y^{-\beta}\right)^{-\alpha}\right]^{\lambda}\left(\frac{\bar{F}(y)}{\bar{F}(x)}\right)^{\gamma_{s}-1}\left\{1-\left(\frac{\bar{F}(y)}{\bar{F}(x)}\right)^{m+1}\right\}^{s-r-1} \frac{f(y)}{\bar{F}(x)} d y .
\end{aligned}
$$

By setting $u=\frac{\bar{F}(y)}{\bar{F}(x)}=\frac{\left[1-\left(1+y^{-\beta}\right)^{-\alpha}\right]^{\lambda}}{\left[1-\left(1+x^{-\beta}\right)^{-\alpha}\right]^{\lambda}}$ from (1.5) in (4.3), we get

$$
\begin{aligned}
E[\xi\{X(s, n, m, k)\} \mid X(r, n, m, k)=x]= & \frac{C_{s-1}\left[1-\left(1+x^{-\beta}\right)^{-\alpha}\right]^{\lambda}}{(s-r-1) ! C_{r-1}(m+1)^{s-r-1}} \\
& \times \int_{0}^{1} u^{\gamma_{s}}\left(1-u^{m+1}\right)^{s-r-1} d u .
\end{aligned}
$$

Again by setting $t=u^{m+1}$ in (4.4), we find that

$$
\begin{gathered}
E[\xi\{X(s, n, m, k)\} \mid X(r, n, m, k)=x] \\
=\frac{C_{s-1}\left[1-\left(1+x^{-\beta}\right)^{-\alpha}\right]^{\lambda}}{(s-r-1) ! C_{r-1}(m+1)^{s-r}} \int_{0}^{1} t^{\frac{\gamma_{s}+1}{m+1}-1}(1-t)^{s-r-1} d t \\
=\frac{C_{s-1}\left[1-\left(1+x^{-\beta}\right)^{-\alpha}\right]^{\lambda}}{C_{r-1}(m+1)^{s-r}} \frac{\Gamma\left(\frac{k+1}{m+1}+n-s\right)}{\Gamma\left(\frac{k+1}{m+1}+n-r\right)} . \\
=\frac{C_{s-1}\left[1-\left(1+x^{-\beta}\right)^{-\alpha}\right]^{\lambda}}{C_{r-1}} \frac{1}{\prod_{j=1}^{s-r}\left(\gamma_{r+j}+1\right)},
\end{gathered}
$$


where

$$
\frac{C_{s-1}}{C_{r-1}}=\prod_{j=1}^{s-r} \gamma_{r+j}
$$

and hence the necessary part.

To prove the sufficient part, we have from (4.1) and (4.2)

$$
\begin{gathered}
\frac{C_{s-1}}{(s-r-1) ! C_{r-1}(m+1)^{s-r-1}} \int_{x}^{\infty}\left[1-\left(1+y^{-\beta}\right)^{-\alpha}\right]^{\lambda}\left[(\bar{F}(x))^{m+1}-(\bar{F}(y))^{m+1}\right]^{s-r-1} \\
\times[\bar{F}(y)]^{\gamma_{s}-1} f(y) d y=g_{s \mid r}(x)[\bar{F}(x)]^{\gamma_{r+1}}
\end{gathered}
$$

where

$$
g_{s \mid r}(x)=\left[1-\left(1+x^{-\beta}\right)^{-\alpha}\right]^{\lambda} \prod_{j=1}^{s-r}\left(\frac{\gamma_{r+j}}{\gamma_{r+j}+1}\right) .
$$

Differentiating (4.5) both sides with respect to $x$, we get

$$
\begin{gathered}
-\frac{C_{s-1}[\bar{F}(x)]^{m} f(x)}{(s-r-2) ! C_{r-1}(m+1)^{s-r-2}} \int_{x}^{\infty}\left[1-\left(1+y^{-\beta}\right)^{-\alpha}\right]^{\lambda}\left[(\bar{F}(x))^{m+1}-(\bar{F}(y))^{m+1}\right]^{s-r-2} \\
\quad \times[\bar{F}(y)]^{\gamma_{s}-1} f(y) d y=g_{s \mid r}^{\prime}(x)[\bar{F}(x)]^{\gamma_{r+1}}-\gamma_{r+1} g_{s \mid r}(x)[\bar{F}(x)]^{\gamma_{r+1}-1} f(x),
\end{gathered}
$$

or

$$
\begin{aligned}
-\gamma_{r+1} g_{s \mid r+1}(x)[\bar{F}(x)]^{\gamma_{r+2}+m} f(x) & \\
& =g_{s \mid r}^{\prime}(x)[\bar{F}(x)]^{\gamma_{r+1}}-\gamma_{r+1} g_{s \mid r}(x)[\bar{F}(x)]^{\gamma_{r+1}-1} f(x),
\end{aligned}
$$

where

$$
\begin{gathered}
g_{s \mid r}^{\prime}(x)=-\alpha \beta \lambda x^{-\beta-1}\left(1+x^{-\beta}\right)^{-\alpha-1}\left[1-\left(1+x^{-\beta}\right)^{-\alpha}\right]^{\lambda-1} \prod_{j=1}^{s-r}\left(\frac{\gamma_{r+j}}{\gamma_{r+j}+1}\right) ; \\
g_{s \mid r+1}(x)=\left[1-\left(1+x^{-\beta}\right)^{-\alpha}\right]^{\lambda}\left(\frac{\gamma_{r+1}+1}{\gamma_{r+1}}\right) \prod_{j=1}^{s-r}\left(\frac{\gamma_{r+j}}{\gamma_{r+j}+1}\right) .
\end{gathered}
$$


Therefore,

$$
\begin{aligned}
\frac{f(x)}{\bar{F}(x)} & =-\frac{g_{s \mid r}^{\prime}(x)}{\gamma_{r+1}\left[g_{s \mid r+1}(x)-g_{s \mid r}(x)\right]} \\
& =\frac{\alpha \beta \lambda x^{-\beta-1}\left(1+x^{-\beta}\right)^{-\alpha-1}}{\left[1-\left(1+x^{-\beta}\right)^{-\alpha}\right]} .
\end{aligned}
$$

Integrating (4.6) on both the sides with respect to $x$ between $(0, y)$, the sufficiency part is proved.

Remark 4.1. (i) As $m \rightarrow-1, k=1$ in (4.2), we get the characterization results from the Kumaraswamy-Burr III distribution based on upper record values as obtained by Kumar et al. [22].

(ii) Setting $m=0, k=1$ in (4.2), we obtain the characterizing results of the Kumaraswamy-Burr III distribution based on order statistics.

Following theorem contains characterization of this distribution by a recurrence relation for the single moments of gos.

Theorem 4.2. For a positive integer $k$ and $j$ be a non-negative integer, a necessary and sufficient condition for a random variable $X$ to be distributed with $p d f$ given by (1.4) is that

$$
\begin{aligned}
\left(1-\frac{j}{\beta \lambda \gamma_{r}}\right) E\left[X^{j}(r, n, m, k)\right]= & E\left[X^{j}(r-1, n, m, k)\right] \\
& +\frac{j}{\alpha \beta \lambda \gamma_{r}} \sum_{u=2}^{\alpha+1}\left(\begin{array}{c}
\alpha+1 \\
u
\end{array}\right) E\left[X^{j+\beta(1-u)}(r, n, m, k)\right] .
\end{aligned}
$$


Proof. The necessary part follows from (2.1). On the other hand, if the relation in (4.7) is satisfied, then on using (1.2), we have

$$
\begin{aligned}
& \frac{C_{r-1}}{(r-1) !} \int_{0}^{\infty} x^{j}[\bar{F}(x)]^{\gamma_{r}-1} f(x) g_{m}^{r-1}(F(x)) d x \\
& =\frac{C_{r-2}}{(r-2) !} \int_{0}^{\infty} x^{j}[\bar{F}(x)]^{\gamma_{r-1}-1} f(x) g_{m}^{r-2}(F(x)) d x+\frac{j C_{r-1}}{(r-1) ! \beta \lambda \gamma_{r}} \\
& \quad \times \int_{0}^{\infty} x^{j}[\bar{F}(x)]^{\gamma_{r}-1} f(x) g_{m}^{r-1}(F(x)) d x \\
& \quad+\frac{j C_{r-1}}{(r-1) ! \alpha \beta \lambda \gamma_{r}} \sum_{u=2}^{\alpha+1}(\alpha+1) \int_{0}^{\infty} x^{j+\beta(1-u)}[\bar{F}(x)]^{\gamma_{r}-1} f(x) g_{m}^{r-1}(F(x)) d x \\
& \frac{C_{r-1}}{(r-1) ! \gamma_{r}} \int_{0}^{\infty} x^{j}[\bar{F}(x)]^{\gamma_{r}-1} f(x) g_{m}^{r-2}(F(x))\left\{\gamma_{r} g_{m}(F(x))-(r-1)[\bar{F}(x)]^{m+1}\right\} d x \\
& =\frac{j C_{r-1}}{(r-1) ! \beta \lambda \gamma_{r}} \int_{0}^{\infty} x^{j}[\bar{F}(x)]^{\gamma_{r}-1} f(x) g_{m}^{r-1}(F(x)) d x+\frac{j C_{r-1}}{(r-1) ! \alpha \beta \lambda \gamma_{r}} \\
& \quad \times \sum_{u=2}^{\alpha+1}(\alpha+1) \int_{0}^{\infty} x^{j+\beta(1-u)}[\bar{F}(x)]^{\gamma_{r}-1} f(x) g_{m}^{r-1}(F(x)) d x .
\end{aligned}
$$

Let

$$
h(x)=-\frac{1}{\gamma_{r}}[\bar{F}(x)]^{\gamma_{r}} g_{m}^{r-1}(F(x)) .
$$

Differentiating both the sides of (4.8), we get

$$
h^{\prime}(x)=\frac{1}{\gamma_{r}}[\bar{F}(x)]^{\gamma_{r}-1} f(x) g_{m}^{r-2}(F(x))\left\{\gamma_{r} g_{m}(F(x))-(r-1)[\bar{F}(x)]^{m+1}\right\} .
$$


Thus,

$$
\begin{aligned}
\frac{C_{r-1}}{(r-1) !} \int_{0}^{\infty} x^{j} h^{\prime}(x) d x= & \frac{j C_{r-1}}{(r-1) ! \beta \lambda \gamma_{r}} \int_{0}^{\infty} x^{j}[\bar{F}(x)]^{\gamma_{r}-1} f(x) g_{m}^{r-1}(F(x)) d x \\
& +\frac{j C_{r-1}}{(r-1) ! \alpha \beta \lambda \gamma_{r}} \sum_{u=2}^{\alpha+1}\left(\begin{array}{c}
\alpha+1 \\
u
\end{array}\right) \int_{0}^{\infty} x^{j+\beta(1-u)} \\
& \times[\bar{F}(x)]^{\gamma_{r}-1} f(x) g_{m}^{r-1}(F(x)) d x .
\end{aligned}
$$

Integrating left hand side in (4.9) by parts and using the value of $h(x)$ from (4.8), we find that

$$
\begin{gathered}
\frac{j C_{r-1}}{(r-1) ! \gamma_{r}} \int_{0}^{\infty} x^{j-1}[\bar{F}(x)]^{\gamma_{r}-1} g_{m}^{r-1}(F(x))\left\{\bar{F}(x)-\frac{x}{\beta \lambda} f(x)\right. \\
\left.-\frac{1}{\alpha \beta \lambda} \sum_{u=2}^{\alpha+1}\left(\begin{array}{c}
\alpha+1 \\
u
\end{array}\right) x^{\beta(1-u)+1} f(x)\right\} d x=0 .
\end{gathered}
$$

Applying the extension of Müntz-Szász Theorem (see, for example, Hwang and Lin [12]), to (4.10), we get

$$
\alpha \beta \lambda \bar{F}(x)=\left\{\alpha x+\sum_{u=2}^{\alpha+1}\left(\begin{array}{c}
\alpha+1 \\
u
\end{array}\right) x^{\beta(1-u)+1}\right\} f(x),
$$

which proves that $f(x)$ has the form as in (1.8).

\section{References}

[1] A. A. Ahmad and A. M. Fawzy, Recurrence relations for single moments of generalized order statistics from doubly truncated distributions, J. Statist. Plann. Inference 117(2) (2003), 241-249.

DOI: https://doi.org/10.1016/S0378-3758(02)00385-3

[2] M. Ahsanullah, Record Statistics, Nova Science Publishers, New York, 1995.

[3] M. Ahsanullah, Generalized order statistics from exponential distribution, J. Statist. Plann. Inference 85(1-2) (2000), 85-91.

DOI: https://doi.org/10.1016/S0378-3758(99)00068-3 
[4] H. Athar and H. M. Islam, Recurrence relations for single and product moments of generalized order statistics from a general class of distributions, Metron International Journal of Statistics LXII(3) (2004), 327-337.

[5] M. Bieniek and D. Szynal, Characterizations of distributions via linearity of regression of generalized order statistics, Metrika 58(3) (2003), 259-271.

DOI: https://doi.org/10.1007/s001840300263

[6] E. Cramer and U. Kamps, Relations for expectations of functions of generalized order statistics, J. Statist. Plann. Inference 89(1-2) (2000), 79-89.

DOI: https://doi.org/10.1016/S0378-3758(00)00074-4

[7] E. Cramer, U. Kamps and C. Keseling, Characterizations via linear regression of ordered random variables: A unifying approach, Comm. Statist. Theory Methods 33(12) (2004), 2885-2911.

DOI: https://doi.org/10.1081/STA-200038832

[8] H. A. David and H. N. Nagaraja, Order Statistics, John Wiley, New York, 2003.

[9] W. Dziubdziela and B. Kopociński, Limiting properties of the $k$-th record values, Appl. Math. (Warsaw) 15(2) (1976), 187-190.

DOI: https://doi.org/10.4064/am-15-2-187-190

[10] M. Habibullah and M. Ahsanullah, Estimation of parameters of a Pareto distribution by generalized order statistics, Comm. Statist. Theory Methods 29(7) (2000), 1597-1609.

DOI: https://doi.org/10.1080/03610920008832567

[11] S. Huang and B. O. Oluyede, Exponentiated Kumaraswamy-Dagum distribution with applications to income and lifetime data, Journal of Statistical Distribution and Applications 1(1) Article 8 (2014), 1-20.

DOI: https://doi.org/10.1186/2195-5832-1-8

[12] J. S. Hwang and G. D. Lin, On a generalized moments problem II, Proc. Amer. Math. Soc. 91(4) (1984), 577-580.

DOI: https://doi.org/10.1090/S0002-9939-1984-0746093-4

[13] U. Kamps, A Concept of Generalized Order Statistics, B. G. Teubner Stuttgart, Germany, 1995.

[14] U. Kamps and E. Cramer, On distribution of generalized order statistics, Statistics 35(3) (2001), 269-280.

DOI: https://doi.org/10.1080/02331880108802736

[15] U. Kamps and U. Gather, Characteristic properties of generalized order statistics from exponential distributions, Applicationes Mathematicae 24(4) (1997), 383-391.

DOI: https://doi.org/10.4064/am-24-4-383-391 
[16] C. Keseling, Conditional distributions of generalized order statistics and some characterizations, Metrika 49(1) (1999), 27-40.

DOI: https://doi.org/10.1007/s001840050023

[17] A. H. Khan and A. A. Alzaid, Characterization of distributions through linear regression of non-adjacent generalized order statistics, J. Appl. Statist. Sci. 13 (2004), 123-136.

[18] A. H. Khan, R. U. Khan and M. Yaqub, Characterization of continuous distributions through conditional expectation of function of generalized order statistics, Journal of Applied Probability \& Statistics 1(1) (2006), 115-131.

[19] R. U. Khan and M. A. Khan, Moment properties of generalized order statistics from exponential-Weibull lifetime distribution, Journal of Advanced Statistics 1(3) (2016), 146-155.

DOI: https://dx.doi.org/10.22606/jas.2016.13004

[20] R. U. Khan and B. Zia, Generalized order statistics from doubly truncated linear exponential distribution and a characterization, Journal of Applied Probability \& Statistics 9(1) (2014), 53-65.

[21] R. U. Khan, Z. Anwar and H. Athar, Recurrence relations for single and product moments of generalized order statistics from doubly truncated Weibull distribution, Aligarh J. Statist. 27 (2007), 69-79.

[22] D. Kumar, M. Kumar, J. Saran and N. Jain, The Kumaraswamy-Burr III distribution based on upper record values, American Journal of Mathematical and Management Sciences 36(3) (2017), 205-228.

DOI: https://doi.org/10.1080/01966324.2017.1318099

[23] V. K. Rohatgi and A. K. Md. E. Saleh, A class of distributions connected to order statistics with nonintegral sample size, Comm. Statist. Theory Methods 17(6) (1988), 2005-2012.

DOI: https://doi.org/10.1080/03610928808829728

[24] S. M. Stigler, Fractional order statistics, with applications, J. Amer. Statist. Assoc. 72(359) (1977), 544-550. 